\title{
Addendum et Erratum
}

\section{Ligand Field Distortion Parameters}

\author{
Bryan R. Hollebone and J. C. Donini
}

Theoret. Chim. Acta (Berl.) 39, 33 (1975)

The original publication [1] did not demonstrate the derivations of Eqs. (7) and (8). These are given here for completeness and to correct a coefficient error in the expansion of (7). The ligand field perturbation Hamiltonian can be derived from the spherical harmonic addition theorem and is of form:

$$
V_{G}=\sum_{n=0}^{\infty} \sum_{m=-n}^{n} \frac{4 \pi}{2 n+1} \frac{r_{<}^{n}}{r_{>}^{n+1}} Y_{m_{j}}^{n^{*}} \cdot Y_{m_{i}}^{n}
$$

In a finite group $G$ representing the physical environment this can be rewritten as a sum over the number of fully symmetric $A_{1}$ components projected from the spherical harmonics:

$$
V_{G}=\sum_{n=0}^{\infty} \sum_{\alpha=1}^{Z} \frac{4 \pi}{2 n+1} \frac{r_{>}^{n}}{r_{>}^{n+1}} \alpha A_{1}^{*} \cdot \alpha A_{1}
$$

In the case of $d$ electrons in an octahedral field $n=4, \alpha=1$

$$
V_{O_{h}}=\frac{4 \pi}{9} \frac{Z e^{2} r_{<}^{4}}{r_{>}^{5}} A_{1}^{*} \cdot A_{1}
$$

Expanding the $A_{1}^{*}$ tensor component of an octahedron in terms of spherical harmonics of order 4 yields:

$$
\begin{aligned}
V_{O_{h}}= & \frac{4 \pi}{9} \frac{Z e^{2} r_{\leq}^{4}}{r_{>}^{5}}\left[\sqrt{\frac{7}{12}} Y_{0}^{4}+\sqrt{\frac{5}{24}}\left(Y_{4}^{4}+Y_{-4}^{4}\right)\right] * A_{1} \\
= & \frac{4 \pi}{9} \frac{Z e^{2} \overline{r_{4}}}{a^{5}}\left[\sqrt{\frac{7}{12}}\left(\sqrt{\frac{1}{2 \pi}} \sqrt{\frac{9}{128}}(8(2)+3(4))\right)\right. \\
& \left.+\sqrt{\frac{5}{24}}\left(\sqrt{\frac{1}{2 \pi}} \sqrt{\frac{9}{128}} \sqrt{\frac{35}{2}}(4+4)\right)\right] A_{1}
\end{aligned}
$$


after substitution of ligand positions in a ligand field approximation. On simplification this becomes:

$$
\begin{aligned}
V_{O_{h}} & =\frac{4 \pi Z e^{2} \overline{r_{4}} \sqrt{7} \sqrt{9}}{9 \sqrt{2 \pi} \sqrt{128} \sqrt{12} a^{5}}(48) A_{1} \\
& =\frac{\sqrt{7 \pi}}{24 \sqrt{3}} \frac{Z e^{2} \overline{r^{4}}}{a^{5}}(48) A_{1} \\
& =\frac{2 \sqrt{7 \pi}}{\sqrt{3}} \frac{Z e^{2} \overline{r^{4}}}{a^{5}} A_{1}
\end{aligned}
$$

Expanding the remaining $A_{1}$ representing the one-electron operator this expression yields:

$$
V_{O_{h}}=\frac{2 \sqrt{7 \pi}}{\sqrt{3}} \frac{Z e^{2} r^{4}}{a^{5}}\left[\sqrt{\frac{7}{12}} Y_{0}^{4}+\sqrt{\frac{5}{24}}\left(Y_{4}^{4}+Y_{-4}^{4}\right)\right]
$$

The conventional form of the operator uses an unnormalized linear combination of spherical harmonics and thus:

$$
\begin{aligned}
V_{O_{h}} & =\frac{2 \sqrt{7 \pi}}{\sqrt{3}} \frac{Z e^{2} \overline{r^{4}}}{a^{5}} \sqrt{\frac{7}{12}}\left[Y_{0}^{4}+\sqrt{\frac{5}{14}}\left(Y_{4}^{4}+Y_{-4}^{4}\right)\right] \\
& =\frac{7 \sqrt{\pi}}{3} \frac{Z e^{2} \overline{r^{4}}}{a^{5}}\left[Y_{0}^{4}+\sqrt{\frac{5}{14}}\left(Y_{4}^{4}+Y_{-4}^{4}\right)\right]
\end{aligned}
$$

which is the conventional form. Instead of completing this simplification, the expansion of (4) may be retained during the expansion of the second $A_{1}$ component in (6). Then:

$$
V_{O_{h}}=\frac{4 \pi}{9} \frac{Z e^{2} \overline{r^{4}}}{a^{5}}\left[\sqrt{\frac{7}{12}} Y_{0}^{4}+\sqrt{\frac{5}{24}}\left(Y_{4}^{4}+Y_{-4}^{4}\right)\right]^{*}\left[\sqrt{\frac{7}{12}} Y_{0}^{4}+\sqrt{\frac{5}{24}}\left(Y_{4}^{4}+Y_{-4}^{4}\right)\right]
$$

This simplifies using (1) to:

$$
\begin{aligned}
V_{O_{h}}= & \frac{4 \pi}{9} \frac{Z e^{2} r^{4}}{a^{5}}\left[\frac{7}{12} Y_{0}^{4 *} Y_{0}^{4}+\frac{5}{24}\left(Y_{4}^{* 4} Y_{4}^{4}+Y_{-4}^{4 *} Y_{-4}^{4}\right)\right] \\
V_{O_{h}}= & \frac{4 \pi}{9} \frac{Z e^{2} \overline{r^{4}}}{a^{5}}\left[\frac{1}{\sqrt{2 \pi}} \sqrt{\frac{9}{128}}\left(\frac{7}{12}\right)\left(\frac{8(2)}{a^{5}}+\frac{3(4)}{b^{5}}\right) Y_{0}^{4}\right. \\
& \left.+\frac{1}{\sqrt{2 \pi}} \sqrt{\frac{315}{256}}\left(\frac{5}{24}\right)\left(\frac{4}{b^{5}} Y_{4}^{4}+\frac{4}{b^{5}} Y_{-4}^{4}\right)\right] \\
= & \frac{4 \pi}{9} \frac{Z e^{2} r^{4}}{a^{5}} \frac{1}{\sqrt{2 \pi}} \sqrt{\frac{9}{128}} \sqrt{\frac{7}{12}}\left[\sqrt{\frac{7}{12}\left(\frac{8(2)}{a^{5}}+\frac{3(4)}{b^{5}}\right) Y_{0}^{4}}\right. \\
& \left.+\frac{5 \sqrt{5}}{2 \sqrt{24}}\left(\frac{4}{b^{5}} Y_{4}^{4}+\frac{4}{b^{5}} Y_{-4}^{4}\right)\right] \\
= & \frac{\sqrt{7 \pi}}{24 \sqrt{3}} Z e^{2} \overline{r^{4}}\left[\sqrt{\frac{7}{12}}\left(\frac{16}{a^{5}}+\frac{12}{b^{5}}\right) Y_{0}^{4}+\frac{5 \sqrt{5}}{2 \sqrt{24}}\left(\frac{4}{b^{5}} Y_{4}^{4}+\frac{4}{b^{5}} Y_{-4}^{4}\right)\right]
\end{aligned}
$$

which is what Eq. (7a) of paper should be. 
The reparametrization of Eqs. (7a) and (7b) to yield Eq. (8) is carried out most easily as a comparison of strong and weak field formulations of the tetragonal Hamiltonian.

Let, in the strong field model:

$$
P\left|A_{1 g}\right|_{D_{4 h}}=D Q\left|A_{1 g} 0\right| o_{h}+D T\left|E_{g} 0\right|_{O_{h}}
$$

In a weak field model;

$$
P\left|A_{1 g}\right|_{D_{4 h}}=P_{0}\left|Y_{0}^{4}\right|+\frac{1}{\sqrt{2}} P_{4}\left|\left(Y_{4}^{4}+Y_{-4}^{4}\right)\right|
$$

Equating these two expressions:

$$
\begin{aligned}
D Q \mid & \left.A_{1 g} 0\left|O_{h}+D T\right| E_{g} 0\right|_{O_{h}}=P_{0}\left|Y_{0}^{4}\right|+\frac{1}{\sqrt{2}} P_{4}\left|\left(Y_{4}^{4}+Y_{-4}^{4}\right)\right| \\
= & \sqrt{\frac{7}{12}} D Q\left|Y_{0}^{4}\right|+\sqrt{\frac{5}{24}} D Q\left|\left(Y_{4}^{4}+Y_{-4}^{4}\right)\right| \\
& +\sqrt{\frac{5}{12}} D T\left|Y_{0}^{4}\right|-\sqrt{\frac{7}{24}} D T\left|\left(Y_{4}^{4}+Y_{-4}^{4}\right)\right|
\end{aligned}
$$

by expansion of the totally symmetric linear combinations of $\left|A_{1 g} 0\right|_{O_{h}}$ and the antisymmetric combination $\left|E_{g} 0\right|_{o_{h}}$. Now equating terms of common harmonics:

$$
P_{0}\left|Y_{0}^{4}\right|=\sqrt{\frac{7}{12}} D Q\left|Y_{0}^{4}\right|+\sqrt{\frac{5}{12}} D T\left|Y_{0}^{4}\right|
$$

and

$$
\frac{1}{\sqrt{2}} P_{4}\left|\left(Y_{4}^{4}+Y_{-4}^{4}\right)\right|=\sqrt{\frac{5}{24}} D Q\left|\left(Y_{4}^{4}+Y_{-4}^{4}\right)\right|+\sqrt{\frac{7}{24}} D T\left|\left(Y_{4}^{4}+Y_{-4}^{4}\right)\right|
$$

Solving for $D Q$ and $D T$ :

$$
\begin{aligned}
& D Q=\sqrt{\frac{5}{12}} P_{4}+\sqrt{\frac{7}{12}} P_{0} \\
& D T=\sqrt{\frac{7}{12}} P_{4}+\sqrt{\frac{5}{12}} P_{0}
\end{aligned}
$$

If these expressions are expanded by substitution of ligand positions where

$$
\begin{aligned}
& P_{0}=\frac{4 \pi}{9} Z e^{2} r^{\overline{4}} \sqrt{\frac{1}{2 \pi}} \sqrt{\frac{9}{128}}\left(\frac{16}{a^{5}}+\frac{12}{b^{5}}\right) \\
& P_{4}=\frac{4 \pi}{9} Z e^{2} r^{4} \sqrt{\frac{1}{2 \pi}} \sqrt{\frac{9}{128}} \sqrt{\frac{35}{2}}\left(\frac{4}{b^{5}}+\frac{4}{b^{5}}\right)
\end{aligned}
$$

then substitution yields:

$$
\begin{aligned}
D Q & =\frac{4 \pi}{9} Z e^{2} r^{\overline{4}} \sqrt{\frac{1}{2 \pi}} \sqrt{\frac{9}{128}}\left[\sqrt{\frac{7}{12}}\left(\frac{16}{a^{5}}+\frac{12}{b^{5}}\right)+\sqrt{\frac{5}{24}} \sqrt{\frac{35}{2}}\left(\frac{4}{b^{5}}+\frac{4}{b^{5}}\right)\right] \\
& =\frac{4 \pi}{9} Z e^{2} r^{\overline{4}} \sqrt{\frac{1}{2 \pi}} \sqrt{\frac{9}{128}} \sqrt{\frac{7}{12}}\left[\frac{16}{a^{5}}+\frac{12}{b^{5}}+\frac{20}{b^{5}}\right] \\
& =\frac{4 \pi}{9} Z e^{2} \overline{r^{4}} \sqrt{\frac{1}{2 \pi}} \sqrt{\frac{9}{128}} \sqrt{\frac{7}{12}}(16)\left[\frac{1}{a^{5}}+\frac{2}{b^{5}}\right]
\end{aligned}
$$

which except for $(4 \pi / 9)$ is Eq. ( $8 a)$. 
The reformulation of this equation to (15) and (25) involved two errors in the paper.

Thus by substitution of (12) of the paper into the equation above:

$$
\begin{aligned}
(D Q)_{4}= & \frac{4 \pi}{9} \frac{Z e^{2} r^{4}}{a} \sqrt{\frac{1}{2 \pi}} \sqrt{\frac{9}{128}}\left[\sqrt { \frac { 7 } { 1 2 } } \left(\frac{8\left(n_{\mathrm{A}}\right)(z)}{(a \sec \theta)^{4}}\right.\right. \\
& \left.-\frac{24\left(n_{E}\right)(x)\left(\tan ^{2} \theta\right)}{(a \operatorname{cosec} \theta)^{4}}+\frac{3\left(n_{E}\right)(x)}{(a \operatorname{cosec} \theta)^{4}}\right) \\
& \left.+\sqrt{\frac{5}{24}} \sqrt{\frac{35}{2}}\left(\frac{2\left(n_{E}\right)(x)}{(a \operatorname{cosec} \theta)^{4}}\right)\right]
\end{aligned}
$$

in which the $\operatorname{cotan} \theta$ function is replaced by a $\tan \theta$ function, implying projection onto the equatorial plane, and the final term is positive not negative. The simplified form of (15) then becomes:

$$
(D Q)_{4}=\frac{4 \pi}{9} \frac{Z e^{2} \overline{r^{4}}}{a} \sqrt{\frac{1}{2 \pi}} \sqrt{\frac{9}{128}}(16)\left[\frac{(z)}{(a \sec \theta)^{4}}-\frac{(x)}{(a \operatorname{cosec} \theta)^{4}}\right]
$$

After simplification Eq. (15) of the paper becomes:

$$
(D Q)_{4}=\frac{2 \sqrt{7 \pi}}{\sqrt{3}} \frac{Z e^{2} r^{4}}{a}\left[\frac{(z)}{(a \sec \theta)^{4}}-\frac{(x)}{(a \operatorname{cosec} \theta)^{4}}\right]
$$

and $(16)$ is

$$
(D Q)_{4}=\frac{9}{8}\left(\frac{-2 n_{3}}{18}+\frac{n_{4}}{6}\right) \frac{2 \sqrt{7 \pi}}{3 \sqrt{3}} \frac{Z e^{2} r^{4}}{a}\left[\frac{(z)}{(a \sec \theta)^{4}}-\frac{(x)}{(a \operatorname{cosec} \theta)^{4}}\right]
$$

Identical alterations should be made to Eqs. (25) for consistency, the new form of (25) is:

$$
\begin{aligned}
(D Q)_{3}= & \frac{9}{8}\left(\frac{2 n_{3}}{18}-\frac{n_{4}}{6}\right) \frac{Z e^{2} r^{4}}{a} \sqrt{\frac{1}{2 \pi}} \sqrt{\frac{9}{128}}\left[\sqrt{\frac{7}{27}}\left(\frac{\frac{8\left(n_{A}\right)(z)}{(a \sec \theta)^{4}}}{(a \operatorname{cosec} \theta)^{4}}+\frac{3\left(n_{E}\right)(x)}{(a \operatorname{cosec} \theta)^{4}}\right)+\sqrt{\left.\frac{20}{54} \sqrt{35}\left(\frac{2\left(n_{E}\right)(x) \tan \theta}{(a \operatorname{cosec} \theta)^{4}}\right)\right]}\right. \\
& -\frac{24\left(n_{E}\right)(x)\left(\tan ^{2} \theta\right)}{=} \frac{9}{8}\left(\frac{2 n_{3}}{18}-\frac{n_{4}}{6}\right) \frac{4}{9} \frac{\sqrt{7 \pi}}{\sqrt{3}} \frac{Z e^{2} r^{4}}{a}\left[\frac{(z)}{(a \sec \theta)^{4}}-\frac{(x)}{(a \operatorname{cosec} \theta)^{4}}\right]
\end{aligned}
$$

As is clearly apparent, these expansions give the expected:

$$
(D Q)_{3}=-\frac{2}{3}(D Q)_{4}
$$

\section{References}

1. Hollebone, B. R., Donini, J. C.: Theoret. Chim. Acta (Berl.) 39, 33 (1975)

Received May 20, 1976 